\title{
Utility of mycobacterial interspersed repetitive unit typing for differentiating Mycobacterium tuberculosis isolates in Wuhan, China \\ Correspondence \\ Lingxiang Ye \\ yelx2004@163.com \\ Received 13 October 2006 \\ Accepted 10 May 2007 \author{
and Lingxiang $\mathrm{Ye}^{1}$
} \\ Hui Han, ${ }^{1,5}$ Fang Wang, ${ }^{2}$ Yong Xiao, ${ }^{3}$ Yi Ren, ${ }^{3}$ Yanjie Chao, ${ }^{4}$ Aizhen Guo ${ }^{4}$

\author{
${ }^{1}$ Department of Epidemiology, School of Public Health, Huazhong University of Science and \\ Technology, Wuhan 430030, China \\ ${ }^{2}$ Department of Social Medicine, School of Public Health, Huazhong University of Science and \\ Technology, Wuhan 430030, China \\ ${ }^{3}$ Wuhan Tuberculosis Prevention and Treatment Hospital, Wuhan 430030, China \\ ${ }^{4}$ National Key Laboratory of Agricultural Microbiology, Huazhong Agricultural University, \\ Wuhan 430070, China \\ ${ }^{5}$ National Center for Women and Children's Health, China CDC, Beijing 100013, China
}

\begin{abstract}
Mycobacterial interspersed repetitive unit (MIRU) typing has been found to allow rapid, reliable, high-throughput genotyping of Mycobacterium tuberculosis, and may represent a feasible approach to study M. tuberculosis molecular epidemiology. To evaluate the use of MIRU typing in discriminating M. tuberculosis strains, isolates from 105 patients in Wuhan City, China, were genotyped by this method as compared to spoligotyping. MIRU typing identified 55 types that defined 21 clusters and 34 unique isolates. The discriminatory power was high [Hunter-Gaston discriminatory index (HGDI), 0.97]. Spoligotyping showed that 86 (81.9\%) of 105 isolates belonged to the Beijing family genotype. For Beijing family and non-Beijing strains, the discriminatory power of MIRU was high ( $\mathrm{HGDI}, 0.95$ and 0.98, respectively). Among the alleles of the MIRU loci for the Beijing family, only locus 26 was highly discriminative, but for non-Beijing strains, loci 10, 16 and 26 were highly discriminative. MIRU typing is a simple and fast method which may be used for preliminary screening of $M$. tuberculosis isolates in China.
\end{abstract}

\section{INTRODUCTION}

Despite global efforts to combat Mycobacterium tuberculosis, it remains a major public health problem worldwide, especially in developing countries such as China. Infection with $M$. tuberculosis is latent, therefore the methods of classical epidemiology cannot reveal disease prevalence. DNA fingerprinting of M. tuberculosis isolates is a powerful tool for studying the molecular epidemiology of tuberculosis (TB). RFLP analysis based on the variation in copy number of an $M$. tuberculosis-specific insertion sequence, IS6110, is a powerful tool for studying molecular epidemiology, and is considered a 'gold standard' for DNA fingerprinting of M. tuberculosis (van Embden et al., 1993; Kremer et al., 1999). The procedures involved, however, are time-consuming and technically demanding, and require large quantities of DNA. For low-copy-number

Abbreviations: HGDI, Hunter-Gaston discriminatory index; MIRU, mycobacterial interspersed repetitive unit; TB, tuberculosis; VNTR, variable number tandem repeat; WTPTH, Wuhan Tuberculosis Prevention and Treatment Hospital. strains (fewer than five IS6110 copies), it is a less reliable indicator of clonality and therefore a poor predictor of epidemiological relationships (Kamerbeek et al., 1997; Warren et al., 1996). A PCR-based typing method using variable number tandem repeats (VNTRs), which are mycobacterial interspersed repetitive units (MIRUs) in 12 human minisatellite-like regions of the $M$. tuberculosis genome, has been developed and has produced some promising initial results (Supply et al., 2000, 2001). Studies have shown that the discriminatory power of this method is comparable to that of IS6110 RFLP (Kremer et al., 2005). Moreover, MIRU typing has also shown its usefulness in studying the population structure of M. tuberculosis (Sola et al., 2003; Supply et al., 2003). Due to its high resolution, simplicity, sensitivity, high reproducibility and easy interlaboratory comparison (Kanduma et al., 2003; Mazars et al., 2001; Mokrousov et al., 2004; Kam et al., 2005), the 12locus MIRU typing method has been found to be highly suitable for global epidemiological surveillance of TB. In some studies, MIRU typing has even been found to produce more distinct patterns than IS6110 RFLP (Barlow et al., 2001; Cowan et al., 2002; Sun et al., 2004). 
The aim of this study was to investigate the differentiation ability of MIRU typing and spoligotyping. Spoligotyping is the 'gold standard' method for identifying Beijing strains (Bifani et al., 2002; Glynn et al., 2002; Mokrousov et al., 2003), which bear the characteristic nine-spacer reaction on spacers 35-43, and show an absence of hybridization to spacers 1-34 (van Soolingen et al., 1995; Kremer et al., 2004). Therefore, spoligotyping was employed to identify the Beijing genotype before MIRU in this study. This will be helpful for assessing the feasibility of appropriate methods in TB epidemiological studies in China.

\section{METHODS}

Mycobacterial strains and genomic DNA. A total of 105 cases of TB constituted $20 \%$ of the 525 patients during the study period. Samples were collected randomly from in-patients at Wuhan Tuberculosis Prevention and Treatment Hospital (WTPTH) from July to December 2005. Wuhan City is one of the largest in central China. WTPTH is a unique facility for the diagnosis and treatment of $\mathrm{TB}$, and all the TB patients accepted treatment in this hospital. The genomic DNA of the studied strains was isolated according to the recommended method (van Embden et al., 1993). M. tuberculosis H37Rv was used as a control. To test the reproducibility of MIRU typing, we blindly typed 20 isolates in two independent experiments.

Spoligotyping. Spoligotyping was performed with a commercial spoligotyping kit (Isogen Bioscience), according to the instructions supplied by the manufacturer. Briefly, template DNA was amplified with 20 pmol biotinylated DRa primer and DRb primer for 25 cycles of $95{ }^{\circ} \mathrm{C}$ for $1 \mathrm{~min}, 55^{\circ} \mathrm{C}$ for $1 \mathrm{~min}$ and $72{ }^{\circ} \mathrm{C}$ for $30 \mathrm{~s}$. Subsequently, the PCR products were heat-denatured and applied to the blot using Miniblotter MN45 (Immunetics). Following the hybridization, the membrane was incubated with horseradish peroxidase-streptavidin conjugate (Zymed). Hybridization signals were detected by enhanced chemiluminescence (Pierce) with a luminocapture instrument (Kodak Image Station 4000MM). Spoligotyping detected the presence of 43 spacer sequences in the direct repeat region by reversed-line blot hybridization (Kamerbeek et al., 1997). The Beijing family genotype was identified by its unique spoligotyping pattern.

MIRU. MIRU analysis was performed essentially as described by Kwara et al. (2003) with the $2 \times$ Taq PCR MasterMix kit (TIANGEN) for the PCR. The amplification parameters consisted of $5 \mathrm{~min}$ at $94{ }^{\circ} \mathrm{C}$, followed by 40 cycles of $30 \mathrm{~s}$ at $94{ }^{\circ} \mathrm{C}, 30 \mathrm{~s}$ at $60{ }^{\circ} \mathrm{C}$ and $30 \mathrm{~s}$ at $72{ }^{\circ} \mathrm{C}$, with a final extension at $72{ }^{\circ} \mathrm{C}$ for $7 \mathrm{~min}$ in an iCycler iQ Multicolor Real-Time PCR detection system (Bio-Rad). The PCR products were analysed on a $2 \%$ agarose gel. The sizes of the amplicons were estimated by comparing with 50 and 100 bp DNA ladders (TIANGEN). The MIRU copy number per locus was calculated as described by Kwara et al. (2003).

Statistical analysis. The MIRU allelic diversity $(h)$ at each of the 12 loci was calculated by the equation $h=1-\sum x i^{2}[n /(n-1)]$, where $x i$ is the frequency of the $i$ th allele at the locus, and $n$ is the number of isolates (Graur \& $\mathrm{Li}, 2000$ ). The Hunter-Gaston discriminatory index (HGDI) (Hunter \& Gaston, 1988) was used as a numerical index for MIRU discriminatory power. HGDI was calculated by using the formula

$\mathrm{HGDI}=1-\left[\frac{1}{N(N-1)} \sum_{J=1}^{S} n_{j}\left(n_{j}-1\right)\right]$ where $N$ is the total number of strains in the typing scheme, $s$ is the total number of different MIRU-VNTR patterns, and $n_{j}$ is the number of strains belonging to the $j$ th pattern.

\section{RESULTS AND DISCUSSION}

\section{Reproducibility of MIRU typing}

In order to check the reproducibility of MIRU typing, we performed two independent blind typing tests with 20 isolates. The results were identical between the two tests, which indicates that MIRU typing had $100 \%$ reproducibility under our conditions.

\section{Genetic diversity of M. tuberculosis isolates in Wuhan}

Using spoligotyping and MIRU typing, we analysed 105 M. tuberculosis isolates from 105 patients. The digital result of the Beijing family spoligotyping genotype is 000000000003771, as described by Dale et al. (2001). Spoligotyping identified 17 types that defined three clusters (91 isolates) and 14 unique isolates. The discriminatory power was 0.33 (HGDI, 0.33). The largest cluster was Beijing family isolates ( 86 isolates, $81.9 \%$ ), which was dominant in Wuhan City. The others were non-Beijing isolates (19 isolates), which defined 13 clusters (HGDI, 0.96).

MIRU typing identified 55 types that defined 21 clusters and 34 unique isolates (HGDI, 0.970; Table 1). Forty types of Beijing family isolates (86 isolates) were identified by MIRU; these defined 17 clusters and 23 unique isolates, with the HGDI being 0.95. The largest cluster was 22322517-3533, with 14 members, followed by 2233-2517-3533, 2223-2517-3533 and 2222-1417-3533, with 11, 4 and 4 members, respectively. The non-Beijing isolates (19 isolates) defined 15 types, 4 clusters and 11 unique isolates (HGDI, 0.98). Members of the Beijing family constitute a homogeneous group of $M$. tuberculosis, as demonstrated by their highly similar RFLP patterns and conserved familyspecific spoligotyping pattern. This homogeneity was also observed in MIRU typing. For non-Beijing isolates, the HGDI for MIRU and spoligotyping was 0.98 and 0.96 , respectively, and the difference between the values was small. However, for Beijing family isolates, the HGDI was 0.95 and 0 , respectively, and the difference was large.

\section{Allelic diversity of the MIRU loci}

Table 2 shows the allelic diversity of the 12 MIRU loci, based on this collection of samples. Loci 10, 16 and 26 were highly discriminative $(\geqslant 0.6)$, loci 23,31 and 40 were moderately discriminative $(\geqslant 0.3)$, and loci $2,4,20,24,27$ and 39 were poorly discriminative $(<0.3)$ when ranked according to Sola et al. (2003). There were some differences between Beijing family and non-Beijing isolates. For Beijing family isolates, only locus 26 was highly discriminative, which supports previous findings (Mokrousov et al., 2004; 
Table 1. MIRU and spoligotyping cluster for $105 \mathrm{M}$. tuberculosis isolates

\begin{tabular}{|c|c|c|c|c|c|}
\hline MIRU pattern ${ }^{*}$ & No. of isolates & Spoligotyping pattern & MIRU pattern ${ }^{\star}$ & No. of isolates & Spoligotyping pattern \\
\hline $2232-2517-3533$ & 14 & 000000000003771 & $2212-2517-3533$ & 1 & 000000000003771 \\
\hline $2233-2517-3533$ & 11 & 000000000003771 & $2221-2511-3522$ & 1 & 767777777760731 \\
\hline $2222-1417-3533$ & 4 & 000000000003771 & $2222-2515-3533$ & 1 & 000000000003771 \\
\hline $2222-2518-3533$ & 3 & 000000000003771 & $2222-2516-3324$ & 1 & 757777777760771 \\
\hline $2232-2516-3533$ & 3 & 000000000003771 & $2222-2516-3533$ & 1 & 000000000003771 \\
\hline $2233-2518-3533$ & 3 & 000000000003771 & $2223-2516-3222$ & 1 & 577000777760771 \\
\hline $2133-2518-3533$ & 2 & 000000000003771 & $2223-2517-3424$ & 1 & 000000000003771 \\
\hline $2212-2417-3533$ & 2 & 000000000003771 & $2223-2517-3433$ & 1 & 000000000003771 \\
\hline $2222-1417-2533$ & 2 & 000000000003771 & $2223-2616-3323$ & 1 & 577777777760731 \\
\hline $2222-1418-3533$ & 2 & 000000000003771 & $2224-1417-3323$ & 1 & 577777770060771 \\
\hline $2222-2513-3533$ & 2 & 000000000003771 & $2232-1417-3533$ & 1 & 000000000003771 \\
\hline 2241-2411-3222 & & 777737777760771 & & & \\
\hline $2241-2511-3322$ & 2 & 777777777760700 & $2232-2518-3433$ & 1 & 000000000003771 \\
\hline \multirow[t]{2}{*}{ 2412-2514-2322 } & 2 & 777757777760771 & $2233-2516-2534$ & 1 & 000000000003771 \\
\hline & & 767777777760731 & & & \\
\hline \multirow[t]{2}{*}{ 2422-2515-2322 } & 2 & 777757777760731 & $2233-2517-3433$ & 1 & 000000000003771 \\
\hline & & 767757777760771 & & & \\
\hline $2233-25110-3533$ & 2 & 000000000003771 & $2233-2518-2531$ & 1 & 000000000003771 \\
\hline $2121-1517-3533$ & 1 & 000000000003771 & $2233-2616-3533$ & 1 & 000000000003771 \\
\hline $2141-1411-3222$ & 1 & 777777777760771 & $2234-2517-3333$ & 1 & 000000000003771 \\
\hline $2212-1417-3333$ & 1 & 000000000003771 & $2234-2518-3233$ & 1 & 000000000003771 \\
\hline $2212-2315-3322$ & 1 & 676763777777600 & $2243-2516-3533$ & 1 & 000000000003771 \\
\hline
\end{tabular}

${ }^{\star}$ Order of MIRU loci: 2, 4, 10, 16, 20, 23, 24, 26, 27, 31, 39, 40.

Nikolayevskyy et al., 2006), loci 10 and 16 were moderately discriminative, and the others were poorly discriminative. Only $25 \%$ of 12 loci were more than moderately discriminative. However, for non-Beijing isolates, loci 10, 16 and 26 were highly discriminative, loci 4, 23, 27, 31 and 40 were moderately discriminative, and the others were poorly discriminative. About $66.7 \%$ of 12 loci had an allelic diversity greater than 0.3 . The difference was significant $(P=0.041)$. This observation implied a relatively greater diversity of genetic makeup of the non-Beijing strains.
In this study, we described the use of MIRU typing as compared with spoligotyping of $M$. tuberculosis isolates. The discriminatory power (HGDI) for MIRU typing and spoligotyping was 0.97 and 0.33 , respectively. It was found that the discriminatory power of the two methods for 19 non-Beijing isolates was almost equal (HGDI, 0.98 and 0.96). This finding was different from those of other studies (Sola et al., 2003). The small number of non-Beijing isolates might be the reason for this difference. In our study, there was $100 \%$ reproducibility in MIRU typing.

Table 2. Allelic diversity of each MIRU locus

\begin{tabular}{|c|c|c|c|c|c|c|c|c|c|c|c|c|}
\hline \multirow[t]{2}{*}{ Alleles ${ }^{\star}$} & \multicolumn{12}{|c|}{ No. of isolates with MIRU locus } \\
\hline & 2 & 4 & 10 & 16 & 20 & 23 & 24 & 26 & 27 & 31 & 39 & 40 \\
\hline$H_{\mathrm{B}}$ & 0 & 0.08 & 0.52 & 0.55 & 0.25 & 0.25 & 0 & 0.60 & 0.08 & 0.23 & 0.03 & 0.23 \\
\hline$H_{\mathrm{N}-\mathrm{B}}$ & 0 & 0.38 & 0.70 & 0.69 & 0.14 & 0.59 & 0 & 0.76 & 0.35 & 0.59 & 0.14 & 0.39 \\
\hline
\end{tabular}

${ }^{\star} H_{\mathrm{T}}$, allelic diversity of 105 M. tuberculosis isolates; $H_{\mathrm{B}}$, allelic diversity of 86 Beijing family isolates; $H_{\mathrm{N}-\mathrm{B}}$, allelic diversity of 19 non-Beijing strains. 
According to the reports by Kremer et al. (1999) and Supply et al. (2000), MIRU typing was also found to be $100 \%$ reproducible, sensitive and specific for M. tuberculosis isolates, a performance that has not been achieved by any other typing method tested under the same conditions.

Most Beijing strains are prevalent in China, where the TB disease burden is high and resources are limited, therefore only an affordable method should be used for typing. This means that efforts must be increased to improve the methodology and cut down costs. MIRU typing based on a capillary electrophoresis system offers convenience, automation, a short turnaround time, high throughput and perfect reproducibility, and may be the method of choice for $M$. tuberculosis strain typing in this country.

\section{ACKNOWLEDGEMENTS}

We thank Chen Jun, Wang Fei, Shen Gang, and all technical staff for their assistance with bacterial strains and electrophoresis. We also thank the Director of WTPTH, Fu Zhijun, for permission to publish this paper.

\section{REFERENCES}

Barlow, R. E., Gascoyne-Binzi, D. M., Gillespie, S. H., Dickens, A., Qamer, S. \& Hawkey, P. M. (2001). Comparison of variable number tandem repeats and IS6110-restriction fragment length polymorphism analyses for discrimination of high- and low-copy-number IS6110 Mycobacterium tuberculosis isolates. J Clin Microbiol 39, 2453-2457.

Bifani, P. J., Mathema, B., Kurepina, N. E. \& Kreiswirth, B. N. (2002). Global dissemination of the Mycobacterium tuberculosis W-Beijing family strains. Trends Microbiol 10, 45-52.

Cowan, L. S., Mosher, L., Diem, L., Massey, J. P. \& Crawford, J. T. (2002). Variable-number tandem repeat typing of Mycobacterium tuberculosis isolates with low copy numbers of IS6110 by using mycobacterial interspersed repetitive units. J Clin Microbiol 40, 1592-1602.

Dale, J. W., Brittain, D., Cataldi, A. A., Cousins, D., Crawford, J. T., Driscoll, J., Heersma, H., Lillebaek, T., Quitugua, T. \& other authors (2001). Spacer oligonucleotide typing of bacteria of the Mycobacterium tuberculosis complex: recommendations for standardised nomenclature. Int J Tuberc Lung Dis 5, 216-219.

Glynn, J. R., Whiteley, J., Bifani, P. J., Kremer, K. \& van Soolingen, D. (2002). Worldwide occurrence of Beijing/W strains of Mycobacterium tuberculosis: a systematic review. Emerg Infect Dis 8, 843-849.

Graur, D. \& Li, W.-H. (editors) (2000). Dynamics of genes in populations. In Fundamentals of Molecular Evolution, p. 58. Sunderland, MA: Sinauer Associates.

Hunter, P. R. \& Gaston, M. A. (1988). Numerical index of the discriminatory ability of typing systems: an application of Simpson's index of diversity. J Clin Microbiol 26, 2465-2466.

Kam, K. M., Yip, C. W., Tse, L. W., Wong, K. L., Lam, T. K., Kremer, K., $\mathrm{Au}$, B. K. \& van Soolingen, D. (2005). Utility of mycobacterial interspersed repetitive unit typing for differentiating multidrugresistant Mycobacterium tuberculosis isolates of the Beijing family. J Clin Microbiol 43, 306-313.
Kamerbeek, J., Schouls, L., Kolk, A., van Agterveld, M., van Soolingen, D., Kuijper, S., Bunschoten, A., Molhuizen, H., Shaw, R. \& other authors (1997). Simultaneous detection and strain differentiation of Mycobacterium tuberculosis for diagnosis and epidemiology. J Clin Microbiol 35, 907-914.

Kanduma, E., McHugh, T. D. \& Gillespie, S. H. (2003). Molecular methods for Mycobacterium tuberculosis strain typing: a users guide. J Appl Microbiol 94, 781-791.

Kremer, K., van Soolingen, D., Frothingham, R., Haas, W. H., Hermans, P. W., Martin, C., Palittapongarnpim, P., Plikaytis, B. B., Riley, L. W. \& other authors (1999). Comparison of methods based on different molecular epidemiological markers for typing of Mycobacterium tuberculosis complex strains: interlaboratory study of discriminatory power and reproducibility. J Clin Microbiol 37, 2607-2618.

Kremer, K., Glynn, J. R., Lillebaek, T., Niemann, S., Kurepina, N. E., Kreiswirth, B. N., Bifani, P. J. \& van Soolingen, D. (2004). Definition of the Beijing/W lineage of Mycobacterium tuberculosis on the basis of genetic markers. J Clin Microbiol 42, 4040-4049.

Kremer, K., Arnold, C., Cataldi, A., Gutierrez, M. C., Haas, W. H., Panaiotov, S., Skuce, R. A., Supply, P., van der Zanden, A. G. \& van Soolingen, D. (2005). Discriminatory power and reproducibility of novel DNA typing methods for Mycobacterium tuberculosis complex strains. J Clin Microbiol 43, 5628-5638.

Kwara, A., Schiro, R., Cowan, L., Hyslop, N. E., Wiser, M. F., Roahen Harrison, S., Kissinger, P., Diem, L. \& Crawford, J. T. (2003). Evaluation of the epidemiologic utility of secondary typing methods for differentiation of Mycobacterium tuberculosis isolates. J Clin Microbiol 41, 2683-2685.

Mazars, E., Lesjean, S., Banuls, A. L., Gilbert, M., Vincent, V., Gicquel, B., Tibayrenc, M., Locht, C. \& Supply, P. (2001). High-resolution minisatellite-based typing as a portable approach to global analysis of Mycobacterium tuberculosis molecular epidemiology. Proc Natl Acad Sci U S A 98, 1901-1906.

Mokrousov, I., Otten, T., Vyazovaya, A., Limeschenko, E., Filipenko, M. L., Sola, C., Rastogi, N., Steklova, L., Vyshnevskiy, B. \& Narvskaya, O. (2003). PCR-based methodology for detecting multidrug-resistant strains of Mycobacterium tuberculosis Beijing family circulating in Russia. Eur J Clin Microbiol Infect Dis 22, 342-348.

Mokrousov, I., Narvskaya, O., Limeschenko, E., Vyazovaya, A., Otten, T. \& Vyshnevskiy, B. (2004). Analysis of the allelic diversity of the mycobacterial interspersed repetitive units in Mycobacterium tuberculosis strains of the Beijing family. Practical implications and evolutionary considerations. J Clin Microbiol 42, 2438-2444.

Nikolayevskyy, V., Gopaul, K., Brown, T., Fedorin, I. \& Drobniewski, F. (2006). Differentiation of tuberculosis strains in a population with mainly Beijing-family strains. Emerg Infect Dis 12, 1406-1413.

Sola, C., Filliol, I., Legrand, E., Lesjean, S., Locht, C., Supply, P. \& Rastogi, N. (2003). Genotyping of the Mycobacterium tuberculosis complex using MIRUs: association with VNTR and spoligotyping for molecular epidemiology and evolutionary genetics. Infect Genet Evol 3, 125-133.

Sun, Y. J., Bellamy, R., Lee, A. G., Ng, S. T., Ravindran, S., Wong, S. Y., Locht, C., Supply, P. \& Paton, N. I. (2004). Use of mycobacterial interspersed repetitive unit-variable-number tandem repeat typing to examine genetic diversity of Mycobacterium tuberculosis in Singapore. J Clin Microbiol 42, 1986-1993.

Supply, P., Mazars, E., Lesjean, S., Vincent, V., Gicquel, B. \& Locht, C. (2000). Variable human minisatellite-like regions in the Mycobacterium tuberculosis genome. Mol Microbiol 36, 762-771.

Supply, P., Lesjean, S., Savine, E., Kremer, K., van Soolingen, D. \& Locht, C. (2001). Automated high-throughput genotyping for study of global epidemiology of Mycobacterium tuberculosis based on 
mycobacterial interspersed repetitive units. J Clin Microbiol 39, 3563-3571.

Supply, P., Warren, R. M., Banuls, A. L., Lesjean, S., van der Spuy, G. D., Lewis, L. A., Tibayrenc, M., van Helden, P. D. \& Locht, C. (2003). Linkage disequilibrium between minisatellite loci supports clonal evolution of Mycobacterium tuberculosis in a high tuberculosis incidence area. Mol Microbiol 47, 529-538.

van Embden, J. D. A., Cave, M. D., Crawford, J. T., Dale, J. W., Eisenach, K. D., Gicquel, B., Hermans, P., Martin, C., McAdam, R. \& other authors (1993). Strain identification of Mycobacterium tuberculosis by DNA fingerprinting: recommendations for a standardized methodology. J Clin Microbiol 31, 406-409.

van Soolingen, D., Qian, L., de Haas, P. E., Douglas, J. T., Traore, H., Portaels, F., Qing, H. Z., Enkhsaikan, D., Nymadawa, P. \& van Embden, J. D. (1995). Predominance of a single genotype of Mycobacterium tuberculosis in countries of east Asia. J Clin Microbiol 33, 3234-3238.

Warren, R., Richardson, M., Sampson, S., Hauman, J. H., Beyers, N., Donald, P. R. \& van Helden, P. D. (1996). Genotyping of Mycobacterium tuberculosis with additional markers enhances accuracy in epidemiological studies. J Clin Microbiol 34, 2219-2224. 